\title{
Spatial summation of chemical irritation and itch produced by topical application of capsaicin
}

\author{
BARRY G. GREEN \\ Monell Chemical Senses Center, Philadelphia, Pennsylvania
}

\begin{abstract}
The effect of increasing the total area of stimulation on the sensations of irritation produced by topical application of capsaicin was studied in two experiments. In the first experiment, stimulus area was varied by changing the size of filter paper disks on which capsaicin was delivered to the skin of the forearm. Subjects rated the intensity and quality of the cutaneous sensations over a 15-min period. Increasing stimulus area by a factor of 15 resulted in a relatively modest increase in the peak perceived intensity of irritation, a shortening of the latency to the onset of irritation, and shifts in the frequency of reports of sensations of itching and stinging/pricking. However, itch was the most frequently reported sensation regardless of stimulus size. In Experiment 2, stimulus area was manipulated by varying the number of stimuli applied to the skin. Despite a smaller difference in total stimulus areas (9-fold vs. 15-fold), the difference in perceived irritation was more pronounced than it was in Experiment 1 and reached statistical significance. It is therefore concluded that spatial summation does occur in the afferent system or systems responsible for the perception of capsaicin on the skin. This result is consistent with previous reports of summation at the threshold for heat pain and constitutes new information about the spatial integration of pruritic stimulation.
\end{abstract}

Spatial summation is a common property of the cutaneous senses. Enlarging the area of stimulation has been shown to increase the sensitivity of the skin to vibration (e.g., Craig, 1966, 1968; Verrillo, 1966), warmth (e.g., Banks, 1976; Stevens \& Marks, 1971), cold (Stevens \& Marks, 1979), and taste (Hara, 1955; McBurney, 1969; Smith, 1971). Although the sense of pain was for a while believed to be an exception among the skin senses (Hardy, Wolff, \& Goodell, 1940), the majority of evidence now suggests that the thresholds for pain induced by heating (Greene \& Hardy, 1958; Kojo \& Pertovaara, 1987; Machet-Pietropaoli \& Chery-Croze, 1979) and by cooling (Machet-Pietropaoli \& Chery-Croze, 1979) also vary inversely with the size of the stimulus. However, most studies of spatial summation in the pain sense have shown that summation in that modality may be less robust than in the other skin senses. Stevens and Marks (1971) noted that the relatively modest summation that had been found for heat pain was consistent with the requirements of a sensory system whose primary function was to signal the occurrence of potentially harmful stimulation, regardless of its spatial extent.

Studies of spatial summation of noxious stimulation have typically employed thermal stimuli. Capsaicin, the pungent compound in chili peppers, offers another form of stimulation with which to measure spatial integration in nociception. It is well established that capsaicin stimulates

This research was supported in part by National Institutes of Health Grant ES04356. The author thanks Linda Flammer for her help in carrying out the experiments and data analyses in this study. Correspondence may be sent to Barry G. Green, Monell Chemical Senses Center, 3500 Market Street, Philadelphia, PA 19104. cutaneous nociceptive afferents (e.g., Adriaensen, Gybels, Handwerker, \& van Hees, 1980; Kenins, 1982; Konietzny \& Hensel, 1983; Szolcsányi, 1977, 1987; Szolcsányi, Anton, Reeh, \& Handwerker, 1988; Torebjörk, 1974). Although the nerve fibers that capsaicin is known to affect (c-polymodal nociceptors and A-delta mechano-heat nociceptors) also respond to heat, there are basic differences in the sensory effects of chemical and thermal stimulation. In particular, a painful temperature stimulus unavoidably produces activity in thermal afferents as well as in nociceptors, especially along the temperature gradient at the circumference of the stimulus. It is therefore likely that the temperature senses (which exhibit abundant summation) contribute either intensity information, quality information, or both to sensations of thermal pain. Coactivation of thermoreceptors and nociceptors also occurs at temperatures a few degrees below the threshold for pain, because some heat-sensitive c-polymodal nociceptors have thermal thresholds in the non-noxious range (Beitel \& Dubner, 1976; van Hees \& Gybels, 1981).

In contrast, despite capsaicin's apparent ability to stimulate warmth-sensitive neurons in the CNS (Nakayama, Suzuki, Ishikawa, \& Nishio, 1978; Szolcsányi, 1982), it has not yet been shown conclusively that the compound stimulates cutaneous warm fibers in significant numbers. A study that recorded the sensation qualities produced by topical application of capsaicin on hairy skin found that sensations of warmth were rarely reported (Green \& Flammer, 1988). The sensation induced by capsaicin applied topically was, however, characterized by burning, itching, and stinging. In addition to constituting indirect evidence that capsaicin does not strongly stimulate the warmth sense, these sensation qualities also raise the pos- 
sibility that the chemical stimulates afferent fibers other than those typically activated by changes in temperature (e.g., specific "itch" fibers, if they exist; see Simone, Ngeow, Putterman, \& Lamotte, 1987; Tuckett, 1982; Tuckett \& Wei, 1987). Evidence from studies in both humans and monkeys indicate that intradermal injection of capsaicin stimulates c-polymodal afferents much less vigorously than does more superficial administration, yet intense burning sensations can result from both modes of application (Simone, Ngeow, Whitehouse, et al., 1987). The latter observation leaves open the possibility that one or more cutaneous chemoreceptors exist that have not yet been identified (see Lamotte, Simone, Baumann, Shain, \& Alreja, 1988; Meyer \& Campbell, 1988).

There are two studies already in the literature that indicate that chemical irritants produce some degree of spatial summation. One study from this laboratory (Green, 1988) demonstrated that when the total area of lingual tissue exposed to a solution of ethanol and water was increased, so too was the intensity of the sensations of irritation produced by the ethanol. In a much earlier study, Keele and Armstrong (1964) reported that the painful sensation produced by the application of acetylcholine to the skin via a blister-base preparation was reduced when the blister base was partially occluded by paraffin. Keele and Armstrong believed that their data reflected a greater amount of summation than had been reported in previous investigations of heat pain. However, those authors did not report the sizes of the areas they stimulated, and their subjects rated the intensity of painful irritation rather than the threshold for pain, as had been done in the thermal experiments.

The two experiments described in this paper represent the first attempts to measure the effect of stimulus size on the intensity of irritation produced by a chemical applied topically to intact, hairy skin. Spatial summation was measured by applying the same concentration of capsaicin to different amounts of skin (i.e., the number of molecules of capsaicin per unit area was held constant as the total area of skin was varied). Under such conditions, summation would be indicated by a trend toward higher perceived intensities as stimulus area increased. The results were consistent with this prediction. However, the amount of summation obtained varied with the manner in which the area of stimulation was increased. In Experiment 1 , enlarging a single stimulus tended to increase the intensity of chemogenic sensations, but the effect of size was not statistically significant; larger stimuli did, however, induce sensations sooner after application of capsaicin than did smaller stimuli. In Experiment 2, increasing the area of stimulation by applying capsaicin to multiple cutaneous sites on the same arm unequivocally demonstrated the occurrence of spatial summation. In both experiments, the dominant sensation quality was itch.

\section{EXPERIMENT 1}

In Experiment 1, measurements were made of the effect of increasing the size of a capsaicin stimulus on both the perceived intensity and the quality of sensations of irritation.

\section{Method}

Subjects. Eight adults ( 5 females and 3 males), between the ages of 21 and 32, were paid to participate. All 8 subjects were laboratory personnel, 4 of whom were experienced in psychophysical tasks.

Stimuli. The stimulus was synthetic capsaicin (8-methyl-nvanillyl-6-noneamide, 97\%; Pfaltz \& Bauer) dissolved in a vehicle of $80 \%$ ethanol and $20 \%$ deionized water. Three different concentrations of capsaicin were used: $0.05 \%, 0.10 \%$, and $0.20 \%$ (by weight). Which concentration was used depended upon the sensitivity of each subject (see below). The solutions were applied to the skin via filter paper disks (Fisher Scientific, porosity equivalent to Whatman No. 1), which had three different surface areas: $4.91,1.27$, and $0.32 \mathrm{~cm}^{2}$. To maintain an equal number of stimulus molecules per unit area of filter paper (and hence of skin), the volume of solution applied to the disks varied in proportion to their size: $0.0700 \mathrm{ml}$ was applied to the largest disk, $0.0181 \mathrm{ml}$ to the intermediate disk, and $0.0045 \mathrm{ml}$ to the smallest disk. These volumes thoroughly wetted the filter paper without causing runoff onto adjacent skin.

Procedure. The subject was seated in a modified dental chair with his/her forearms resting on the arms of the chair such that the volar (ventral) surfaces faced upward. Each subject sat in this manner to enable simultaneous application of the test stimulus and the vehicle to opposite arms. To ensure that each subject was tested under similar ambient and cutaneous conditions, at the start of each session the temperatures of the room and of the subject's forearms were measured using a digital thermocouple thermometer. The mean ambient temperature throughout the study was $25.1^{\circ} \mathrm{C}(S D=1.07)$, and the mean skin temperature was $31.7^{\circ} \mathrm{C}(S D=0.74)$.

The experimenter measured the distance from the broadest part of the subject's forearm to the wrist (along the midline). This distance was recorded to enable stimulation of the same site on both arms throughout the study. Filter paper disks were then placed on the designated sites on both arms, after which the experimenter pipetted either the capsaicin stimulus or the vehicle onto one of the sites. Immediately following application of the solution, a $16-\mathrm{cm}^{2}$ $(4 \mathrm{~cm} \times 4 \mathrm{~cm}$ ) piece of parafilm was placed over the filter paper. A bead of denture adhesive cream (Fixodent, Richardson-Vicks, Inc.) applied to the bottom edge of the parafilm produced an airtight seal with the skin. This method of stimulation prevented significant evaporation of the solutions and avoided generation of cutaneous sensations unrelated to the chemical stimulus.

After applying a solution to one arm, the experimenter pressed a key to initiate a computerized timing program. Thirty seconds later (signaled by a tone), she applied the other solution (either capsaicin or the vehicle) to the second arm and covered it with parafilm.

The saturated filter paper disks were left in contact with both arms for $10 \mathrm{~min}$, after which they (and the parafilm) were removed with forceps. During that $10 \mathrm{~min}$, and for an additional $10 \mathrm{~min}$ thereafter, the subject attended to both arms and reported, at 30-sec intervals (signaled by a tone), if a cutaneous sensation had occurred on either arm. If sensations were perceived on both arms, the subject reported only the arm that had the stronger sensation. The subject was required to (1) state on which arm the sensation was felt, (2) describe its quality, and (3) give a magnitude estimate of total perceived intensity. Reports of sensation quality were based on six descriptors: cold, warm, itch, sting/prick, burn, and pain. These terms had been used previously in studies of cutaneous chemogenic sensations (Green \& Flammer, 1988, 1989). The subjects were encouraged to use as few or as many of the terms as necessary to describe the sensations they perceived on each trial. The thermal descriptors were considered self-explanatory, but written definitions were provided for the remaining terms. Itch was described as "the sensation associated with a desire to scratch"; sting/prick 
was defined as "sharp sensations similar to those produced by an insect bite or a pin-prick, and may be constant or intermittent"; burn was described as "the sensation produced by extreme temperatures or by chemical irritants, which may or may not be associated with a thermal sensation"; and pain was explained as an appropriate label for "any sensation that "hurts."

Following the report of sensation quality, the subject responded with a magnitude estimate of the overall intensity of the sensations. Practice was given in the method of magnitude estimation prior to the first session by having each subject assign numbers to distances the experimenter produced between her hands. The subject then donned headphones and listened to a burst of white noise of moderate intensity to which he/she assigned a magnitude estimate. The noise, which subjects could trigger with a foot switch at any time during the session, served as an intensity standard against which they compared and rated the cutaneous sensations. In succeeding sessions, each subject was reminded of the number he or she had assigned to the noise and was again told to rate the cutaneous sensations relative to the noise and the number associated with it.

The first session of the experiment was used to obtain information about each subject's sensitivity to capsaicin. A solution of $0.05 \%$ capsaicin, applied to the largest filter paper disk, served as the test condition. If the subject reported feeling sensations on at least 10 consecutive 30-sec intervals and identified the correct ("test") arm $75 \%$ of the time or better throughout the entire session, that concentration was considered detectable (this criterion had been used in two similar studies in our laboratory; see Green \& Flammer, $1988,1989)$. If the $0.05 \%$ solution was not detected, the concentration was doubled in the next session, and so on. Once detected, that solution was used for that particular subject throughout the experiment. It turned out that 4 subjects were tested in the experiment proper with $0.05 \%, 3$ with $0.10 \%$, and 1 with $0.20 \%$.

Every subject was tested in a total of nine experimental sessions: the three stimulus sizes were presented three times each, with the order of presentation randomized. The magnitude estimates were standardized across subjects by dividing each subject's numerical rating of the noise stimulus into 10 (an arbitrarily chosen value) then, for each subject, multiplying the ratings of the cutaneous sensations by the resulting quotient.

\section{Results and Discussion}

Figure 1 contains the arithmetic means of the standardized estimates of perceived irritation for the three sizes that were tested. Although the data showed a trend

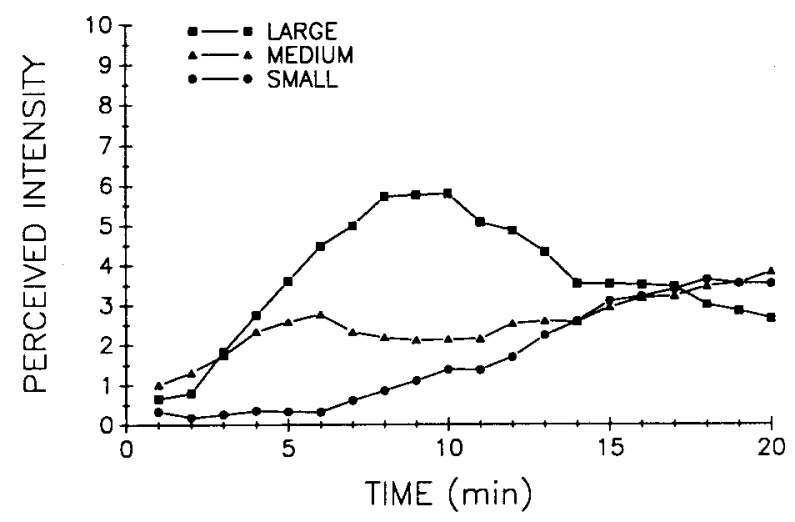

Figure 1. Shown as functions of time of exposure to capsaicin are the perceived intensities of sensations of irritation for the three stimulus sizes tested in Experiment 1 . Large $=4.91 \mathrm{~cm}^{2}$, Medium = $1.27 \mathrm{~cm}^{2}$, and Small $=0.32 \mathrm{~cm}^{2}$. toward higher magnitude estimates as the size of the stimulus increased, a repeated measures ANOVA indicated that the trend was not statistically significant $[F(2,14)=1.49$, $p=.259$ ]. In contrast, the effect of time was significant $[F(19,133)=1.94, p=.016]$, as was the interaction between time and size $[F(38,266)=1.37, p=.003]$.

The absence of a main effect of stimulus size is due in part to the similarity in intensities at the beginning and end of the observation period, when the sensations were growing and declining. The significant interaction between time of exposure and size reflects the differences in the time course of the sensation for the three areas. For example, the latency to the onset of irritation was briefer for the two larger stimuli: The 4.91 - and $1.27-\mathrm{cm}^{2}$ stimuli produced sensations of irritation in the first 1-3 min of exposure, whereas the $0.32 \mathrm{~cm}^{2}$ stimulus produced a gradual growth in irritation only after 6-7 min of exposure. In addition, the shape of the function for the largest stimulus differed from the shapes of the other two functions, exhibiting a clear peak in intensity before declining gradually over the remainder of the observation period. After 16-17 min, sensation was nearly equal for all three stimulus sizes.

Figure 2 contains the data for sensation quality averaged across time of exposure for each subject. Only the three most frequently used descriptors (itch, sting/prick, and burn) are depicted. The data for each of the 8 subjects has been shown to illustrate the individual differences that were observed. It is obvious from the figure that the dominant perceptual quality was itch, followed by sting/prick and burn. Although not depicted in Figure 2, the dominance of itch continued throughout the testing period. The appearance of itch was expected in light of previous work (Green \& Flammer, 1988), but its dominance over other sensation qualities was not. In an earlier study from our laboratory, burn was most frequently used to describe the capsaicin sensation, with sting and prick being approximately as common as itch. Because individual variations in the sensitivity to pruritic stimuli can be dramatic (e.g., Cormia, 1952; Shelley \& Arthur, 1957), the frequency of reports of itch probably depends upon the individuals tested. Overall, however, it is clear that when topically applied (in an ethanol and water vehicle), capsaicin is a pruritic stimulus as well as a simple irritant.

An analysis of the quality data employing the nonparametric Friedman ANOVA revealed that there was a significant effect of size on the frequency of reports of itch $\left[\chi^{2}(2)=10.938, p=.0042\right]$ and sting/prick $\left[\chi^{2}(2)=\right.$ $11.812, p=.0027]$, but not burn. The significant differences reflect trends toward fewer itch responses and more sting/prick responses as stimulus size increased. There were no reports of pain, and reports of warmth were so infrequent that they were not analyzed separately; for all three sizes, sensations of warmth were felt on no more than $10 \%$ of the trials during which any sensations were reported. The paucity of reports of warmth affords additional indirect evidence that, when applied to the forearm, 


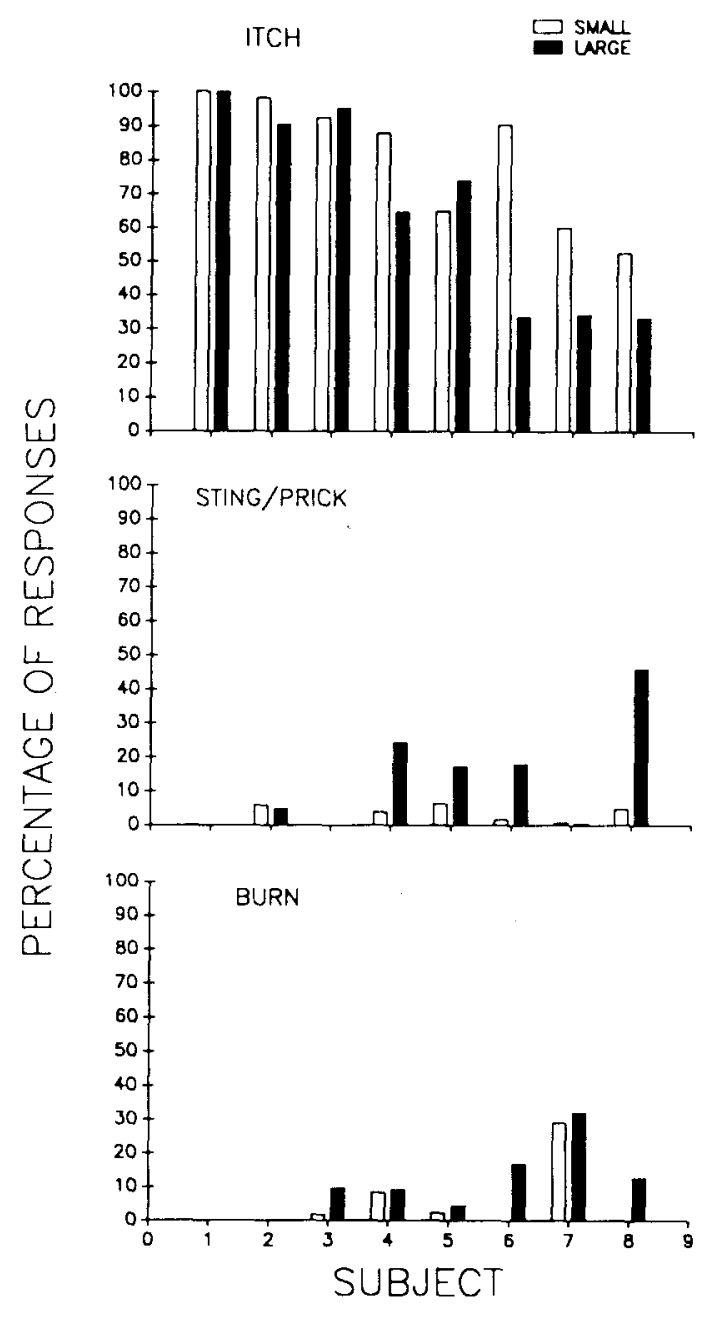

Figure 2. The percentage of reports of itch, sting/prick, and burn given by each of the subjects in response to the small and large stimuli. The subjects have been arranged according to the frequency of reports of sensations of itch. Note the individual differences in both the qualities most frequently reported and the effects of stimulus size.

capsaicin probably does not stimulate cutaneous warm fibers in significant numbers (Green \& Flammer, 1988). Otherwise, the generous spatial summation in the warmth sense would be expected to produce increasingly strong sensations of warmth as the area of stimulation increased.

Overall, the results of Experiment 1 support the hypothesis that area of stimulation has at least a weak effect on the perception of the irritation produced by capsaicin. Despite the absence of a significant main effect of stimulus size, it was found that the temporal course of the sensation depended upon the amount of skin stimulated. The tendency for the sensation to grow more rapidly for the larger stimuli may also be indicative of the combined effects of spatial and temporal summation. Evidence from studies of the relationship between nociceptor activity and sensation in humans suggests that temporal summation occurs in the cutaneous nociceptive system (Adriaensen et al., 1980). Increasing the number of cutaneous units sending impulses into a central integrator should therefore result in an earlier, as well as a more intense, sensation of irritation.

\section{EXPERIMENT 2}

In addition to simply enlarging a single stimulus, the total amount of skin stimulated can also be increased by adding stimuli. It was decided to perform a second experiment that used this alternative approach, in part because the results of Experiment 1 were somewhat equivocal with respect to the effect of size on perceived intensity, and in part because presenting stimuli to multiple sites offered an opportunity to avoid a possible confound that was inherent in the design of Experiment 1. Because the subjects could both see and feel (via both tactile and thermal sensations) the size of the stimuli, it was possible that they had rated the larger stimuli as more intense simply because of their size. Conversely, the amount of summation may have been underestimated if the subjects attempted to compensate for the differences in size. In Experiment 2 , we avoided this potential problem by applying several drops of the chemical stimulus to the skin in rapid succession via micropipette in such a way that the size of the stimulus always appeared the same.

\section{Method}

Subjects. Eight adults $(6$ females and 2 males, average age $=$ 28.8 years) were paid to participate. All subjects were employees of the Monell Center, and 7 of them were experienced in psychophysical tasks.

Stimuli. The stimuli were 0.006 -ml drops of $1.0 \%$ (by weight) synthetic capsaicin (97\%; Pfaltz \& Bauer) dissolved in a vehicle of $80 \%$ ethanol and $20 \%$ water. A much higher concentration of capsaicin was used in Experiment 2 because the stimuli were not covered (which allowed evaporation to occur) and because they were applied as single drops that diffused over an area of skin no larger than approximately $1 \mathrm{~cm}$ in diameter (about $0.8 \mathrm{~cm}^{2}$ ).

Procedure. The physical setup was the same as in Experiment 1. Once again, the temperatures of the skin and the laboratory were recorded at the beginning of each session. Skin temperature averaged $31.6^{\circ} \mathrm{C}(S D=0.77)$ and ambient temperature averaged $23.6^{\circ} \mathrm{C}(S D=0.35)$.

To enable placement of the chemical stimuli on the same location and in the same relationship to one another day after day, a rectangular cardboard template $(2.5 \mathrm{~cm} \times 5.0 \mathrm{~cm})$ was centered on the broadest part of the ventral surface of the forearm such that the template's longest axis was aligned with the long axis of the arm. Individual ink marks were then placed at the four corners of the template, at the midpoint of each of the four sides, and in the middle of the template. With the template removed, these nine marks appeared as three rows of dots oriented transversely across the forearm. The dots in each row were separated from one another by $1.25 \mathrm{~cm}$; the rows were spaced $2.5 \mathrm{~cm}$ apart (longitudinally).

Once the marks had been made, the experimenter pressed a key to initiate a computerized timing program. She then proceeded to apply drops of the stimulus or the vehicle on the marked locations. In one condition, the $1.0 \%$ capsaicin solution was applied to all nine locations; in the other condition, capsaicin was applied only to the middle point in the middle line (i.e., the point in the middle of the array) and the other eight loci received only the vehicle. Application was always in the same spatial sequence, starting with the 
row of dots closest to the subject's elbow and proceeding distally. Five minutes after application of the stimuli had begun, a tone sounded signaling the beginning of the observation period. At that time, the subject was asked to report on the quality and intensity of the sensations he/she felt on the arm. The same descriptors used in Experiment 1 were used again here, and magnitude estimation was used to obtain ratings of the perceived intensity of irritation. Rather than simply specifying the qualities of the sensations they perceived, the subjects were instructed both to name the qualities and to provide magnitude estimates for each of them. This procedure was used in lieu of obtaining a single overall estimate of perceived intensity. If no sensation was detectable during a given observation interval, the subject was instructed to say "zero." Judgments were obtained every $60 \mathrm{sec}$ for a period of $15 \mathrm{~min}(5$ to $19 \mathrm{~min}$ after application of the stimuli began).

The noise stimulus was again used as a standard in the magnitude estimation task, and the group data were standardized in terms of the numbers assigned to the noise in the manner of Experiment 1. The subjects served in four sessions to contribute four observations per time interval for both stimulus conditions, bringing the total number of observations per data point to 32 . The arm tested was alternated across sessions, with half the subjects being tested first with the left arm and half first with the right. The two conditions were counterbalanced across subjects and sessions in the same manner.

\section{Results and Discussion}

Shown in Figure 3 is the overall perceived irritation (i.e., the sum of the ratings across sensation qualities) as a function of time. It is apparent that the sensation produced by stimulating nine cutaneous sites grew to be more intense than the sensation produced by stimulating one cutaneous site. The statistical reliability of this difference was confirmed by a repeated measures ANOVA, which revealed the existence of a significant main effect of stimulus number $[F(1,7)=25.98, p=.0014]$. Also significant was the effect of time of rating $[F(14,98)=$ $7.31, p<.0001]$ and the interaction between stimulus number and time of rating $[F(14,98)=3.92, p<.0001]$. The latter effect derived from both the steeper initial

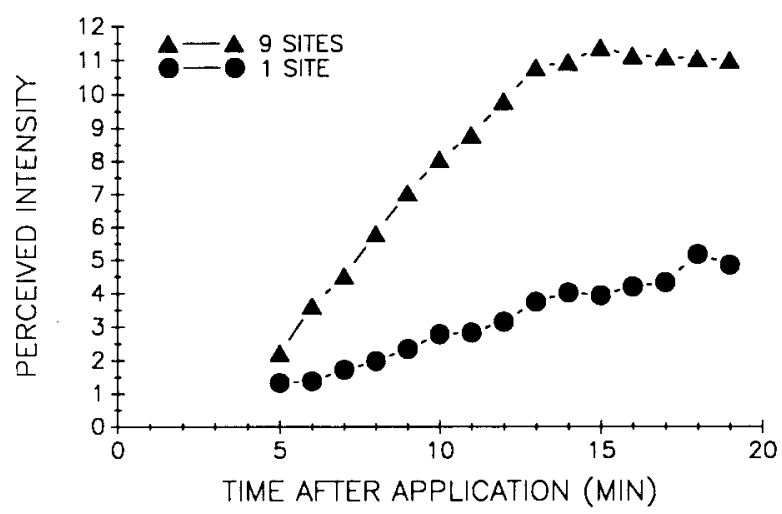

Figure 3. The perceived intensities of sensations of irritation as functions of the time after application of the stimuli for the ninesite (triangles) and one-site (circles) stimuli.

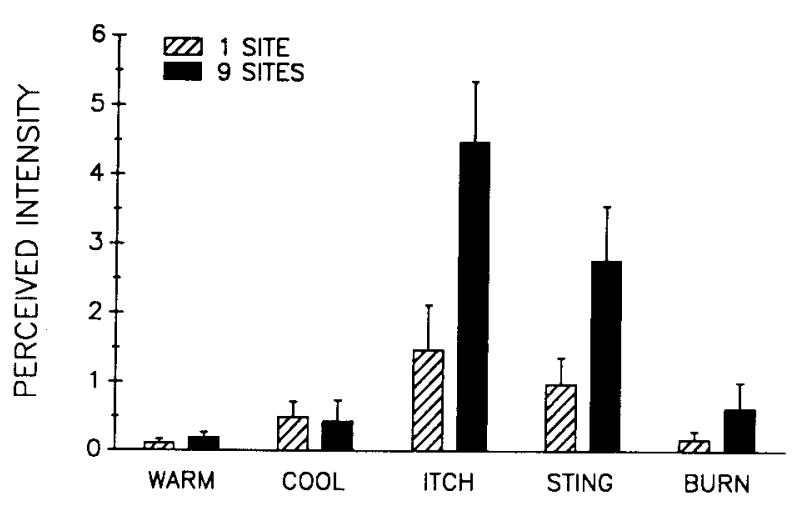

Figure 4. Shown are the perceived intensities reported for the five sensation qualities. The data are the mean intensities collapsed across rating period. Bars indicate standard errors of the means.

rise in sensation intensity and the subsequent leveling-off that occurred for the nine-site stimulus compared with the gradual rise in sensation produced by the single-site stimulus. Linear regressions performed on the data for Minutes 5 through 13 revealed that the slope of the function for nine sites was more than three times steeper than was the slope of the function for one site (1.06 vs. 0.31$)$.

Over the final $5 \mathrm{~min}$ of observation, the total irritation reported for the nine-site stimulus was on average 2.8 times more intense than was the irritation reported for the single-site stimulus. It is not possible to determine precisely how much summation this difference represents, because the rate of growth of perceived irritation as a function of the mass of capsaicin applied to the skin has not been established. That is, it will be necessary to know the slope of the psychophysical function for capsaicin on hairy skin before a prediction can be made as to the change in perceived intensity that should occur when stimulus mass is increased ninefold. In addition, using the sum of the perceived intensities across qualities assumes that the perceptual magnitudes of the qualities add linearly to produce total intensity, which, though reasonable, may not be true. (This assumption did not affect the outcome of the statistical analysis. An ANOVA run on the mean ratings across qualities produced the same pattern of significant effects, which rules out the possibility that the effects were due to increases in the number of qualities reported for each stimulus rather than to increases in the perceived magnitude of the qualities.)

Figure 4 shows the mean perceived intensities for each of the five sensation qualities that were reported (pain was never experienced). The data are collapsed across time. As in Experiment 1, the dominant quality was itch, with sting/prick and burn reliably reported to have lower intensities. The ANOVA revealed a significant main effect of sensation quality $[F(4,28)=9.51, p=.0001]$ and a significant interaction between quality and stimulus size $[F(4,28)=10.27, p<.0001]$. The main effect indicates 
that perceived magnitude varied significantly across qualities, whereas the interaction is probably due most to the absence of an effect of stimulus size on the qualities of warmth and cold. However, a simple effects analysis also showed that stimulus size failed to influence significantly the intensity of burn responses $[F(1,7)=2.32, p=.171]$; only the sensations of sting/prick $[F(1,7)=10.24, p=$ $.015]$ and itch $[F(1,7)=20.94, p=.0026]$ were reliably affected by the number of sites stimulated.

Both the low perceived intensity of sensations of warmth and the absence of a significant effect of stimulus area provide additional evidence that the warmth sense is not strongly affected by topical applications of capsaicin. The somewhat stronger sensations of cold probably do not reflect a larger effect of capsaicin on cold fibers than on warm fibers; reports of cold occurred mostly during the first few minutes after application when the effects of evaporative cooling (due primarily to the ethanol vehicle) were still being felt (see also Green \& Flammer, 1988).

In contrast to the results of Experiment 1, the frequency of reports of itch and sting/prick did not vary significantly between conditions. Reports of itch actually increased slightly when more sites were stimulated $(43.5 \%$ vs. $39 \%)$, whereas sting and/or prick were reported on $32 \%$ of the trials when nine sites were stimulated and on $36.6 \%$ of the trials when one site was stimulated. Sensations of burning were again experienced infrequently, accounting for only $5.8 \%$ and $8.3 \%$ of the reports for the one- and ninesite stimuli.

\section{GENERAL DISCUSSION}

The present study has demonstrated that the size of the stimulus can be important for establishing the time course and intensity of chemogenic irritation on hairy skin. Spatial summation appears to be a characteristic of the sensory system or systems that mediate the response to capsaicin. Because it is known that c-polymodal nociceptors and some A-delta nociceptors respond to capsaicin, the results provide further support for the notion that the pain sense is capable of spatial integration. This conclusion must, however, be tempered: the ubiquitous and strong sensations of itch reported in the present experiments raise the question of whether the results can be generalized to other chemical irritants that produce other sensation qualities. It is arguable from Figures 2 and 4 that we have, in effect, measured spatial summation of itch. Perhaps a stimulus like methyl salicylate, which has been shown to produce virtually pure burning sensations on hairy skin (Green \& Flammer, 1989), would show more or less spatial summation. Such a result would be interesting because it would imply that separate elements of the nociceptive system mediate itch and burn, and that the central mechanisms to which they are connected process spatial information differently.

It is unclear why spatial summation was more pronounced in Experiment 2 than in Experiment 1. Perhaps, as was noted earlier, the fact that the subjects could see and feel the size of the stimuli in Experiment 1 induced a response bias that obscured the amount of summation. On the other hand, the same tendency toward a larger effect of stimulus area for dual-versus single-site stimulation was also present in a study of the spatial and temporal characteristics of ethanol irritation on the tongue (Green, 1988). It is possible, therefore, that the difference in summation found in the present study is real, and that it is indicative of the manner in which spatial integration occurs in the nociceptive system. For example, separating the stimuli in the nine-stimulus complex by a few centimeters may have optimized summation by reducing the influence of opposing lateral inhibitory effects. It is apparent both from our own observations (Green, 1989) and from the phenomenon of counterirritation (e.g., Chung, 1985; Duncker, 1937; Murray \& Weaver, 1975; Parsons \& Goetzl, 1945) that strong inhibitory effects can occur between chemogenic (and other) nociceptive stimuli. The relationship between summation and inhibition, and the influence of stimulus separation on both phenomena, are topics deserving of further study.

Taken together the data from Experiments 1 and 2 indicate that the effect of stimulus size on the quality of chemogenic sensations is neither large nor reliable. It appears that the mere number of afferents stimulated is not a major determinant of perceptual quality. The independence of perceived intensity and sensation quality is most apparent in Figure 4 where the quality "profile" of the capsaicin sensation was the same for both stimuli. Despite finding a significant shift in the frequency of reports of itch and sting/prick in Experiment 1, no substantial change in frequency of reports was observed across areas in Experiment 2 . The latter result is convincing given the pronounced changes in perceived intensity that occurred. It remains possible, however, that a shift in quality does occur when stimulation produced by larger areas approaches painful levels (e.g., sensations of itch may give way to sensations of stinging or burning pain). In the present study, concentrations were adjusted to avoid producing pain with the smallest stimuli, because it was thought that a transition between innocuous irritation and pain might be demonstrated when stimulus size was increased. That this failed to happen does not mean that such an effect cannot occur; indeed, one subject dropped out of the present study because she reported that the sensations produced by the multiple-point stimulus in Experiment 2 were too painful. The same subject did not experience pain when exposed to the smaller stimulus.

A related issue is whether summation also occurs at the threshold for detection of chemical stimuli. Because the concentrations used in both experiments produced relatively weak sensations of irritation, the present data are at least suggestive that it does. However, it will be necessary to measure the detectability of stimuli of different size to confirm or disconfirm this hypothesis.

In addition to providing data on the spatial integration of chemical irritation on the skin, the present study illustrates that chemical irritants are a viable alternative to 
intense thermal stimulation for the investigation of the perceptual characteristics of the nociceptive system. No other type of stimulation has the potential for providing so clear a picture of the perceptual consequences of stimulating this system at levels that do not produce pain. It should become possible to use chemicals as increasingly specific probes of nociceptive function as more is learned about the types of afferent fibers that particular chemicals stimulate.

\section{REFERENCES}

adriaensen, H., Gybels, J., Handwerker, H. O., \& van Hees, J. (1980). Latencies of chemically-evoked discharges in human cutaneous nociceptors and of the concurrent subjective sensations. Neuroscience Letters, 20, 55-59.

Banks, W. P. (1976). Areal and temporal summation in the thermal reaction time. Sensory Processes, 1, 2-13.

Beitel, R. E., \& DUBner, R. (1976). Response of unmyelinated (c) polymodal nociceptors to thermal stimuli applied to the monkey's face. Journal of Neurophysiology, 39, 1160-1175.

CHUng, J. M. (1985). Antinociceptive effects of peripheral nerve stimulation. In M. J. Correia \& A. A. Perachio (Eds.), Contemporary sensory neurobiology (pp. 47-161). New York: Alan R. Liss.

Cormia, F. E. (1952). Experimental histamine pruritus. Journal of Investigative Dermatology, 19, 21-33

CraIG, J. C. (1966). Vibrotactile loudness addition. Perception \& Psychophysics, 1, 185-190.

Craig, J. C. (1968). Vibrotactile spatial summation. Perception \& Psychophysics, 5, 351-354.

DUNCKER, K. (1937). Some preliminary experiments on the mutual influence of pains. Psychologische Forschung, 21, 311-326.

GreeN, B. G. (1988). Spatial and temporal factors in the perception of ethanol irritation on the tongue. Perception \& Psychophysics, 44, 108-116.

GreEN, B. G. (1989). [Inhibitory effects of contralateral chemogenic irritation.] Unpublished raw data.

Green, B. G., \& Flammer, L. N. (1988). Capsaicin as a cutaneous stimulus: Sensitivity and sensory quality on hairy skin. Chemical Senses, 13, 367-384.

Green, B. G., \& Flammer, L. N. (1989). Methyl salicylate as a cutaneous stimulus: A psychophysical analysis. Somatosensory \& Motor Research, 6, 253-274.

GREENE, L. C., \& HARDY, J. D. (1958). Spatial summation of pain. Journal of Applied Physiology, 13, 457-464.

HARA, S. (1955). Interrelationships among stimulus intensity, stimulated area and reaction time in the human gustatory sensation. Bulletin of the Tokyo Medical \& Dental University, 2, 147-158.

HARDY, J. D., WolfF, H. G., \& GoOdelL, H. (1940). Studies on pain. A new method for measuring pain thresholds: Observations on spatial summation of pain. Joumal of Clinical Investigation, 19, 649-657.

KeEle, C. A., \& ARMSTRONG, D. (1964). Substances producing pain and itch. London: Edward Arnold.

KENINS, P. (1982). Responses of single nerve fibers to capsaicin the skin. Neuroscience Letters, 29, 83-88.

Kojo, I., \& Pertovana, A. (1987). The effects of stimulus area and adaptation temperature on warm and heat pain thresholds in man. International Journal of Neuroscience, 32, 875-880.

KonietzNY, F., HenSEL, H. (1983). The effect of capsaicin on the response characteristic of human c-polymodal nociceptors. Journal of Thermal Biology, 8, 213-215.

Lamotte, R. H., Simone, D. A., Baumann, T. K., Shain, C. N., \& ALREJA, M. (1988). Hypothesis for novel classes of chemoreceptors mediating chemogenic pain and itch. In R. Dubner, G. F. Gelhart, \& M. R. Bonds (Eds.), Proceedings of the Vth World Congress on Pain (pp. 529-535). New York: Elsevier.

Machet-Pietropaoli, H., \& Chery-Croze, S. (1979). Spatial summation of thermal pain in humans. Sensory Processes, 3, 183-187.

MCBurNey, D. H. (1969). A note on the relation between area and intensity in taste. Perception \& Psychophysics, 6, 250.

MEYER, R. A., \& CAMPBELL, J. N. (1988). A novel electrophysiological technique for locating cutaneous nociceptive and chemospecific receptors. Brain Research, 441, 81-86.

Murray, F. S., \& Weaver, M. M. (1975). Effects of ipsilateral and contralateral counterirritation on experimentally produced itch in human beings. Journal of Comparative Physiology \& Psychology, 89 , 819-826

Nakayama, T., Suzuki, M., Ishikawa, Y., Nishio, A. (1978). Effects of capsaicin on hypothalamic thermo-sensitive neurons in the rat. Neuroscience Letters, 7, 151-155.

Parsons, C. M., Goetzl, F. R. (1945). Effect of induced pain on pain threshold. Proceedings of the Society for Experimental Biology (NY), 60, 327-329.

Shelley, W. B., ARThur, R. P. (1957). The neurohistology and neurophysiology of the itch sensation in man. Archives of Dermatology, 76, 296-323.

Simone, D. A., Ngeow, J. Y. F., Putterman, G. J., \& Lamotte, R. H. (1987). Hyperalgesia to heat after intradermal injection of capsaicin. Brain Research, 418, 201-203.

Simone, D. A., Ngeow, J. Y. F., Whitehouse, J., BecerraCabal, L., Putterman, G. J., \& Lamotte, R. H. (1987). The magnitude and duration of itch produced by intracutaneous injections of histamine. Somatosensory Research, 5, 81-92.

SMITH, D. V. (1971). Taste intensity as a function of area of concentration: Differentiation between compounds. Journal of Experimental Psychology, 87, 163-171.

Stevens, J. C., MARKS, L. E. (1971). Spatial summation and the dynamics of warmth sensation. Perception \& Psychophysics, 9, 391-398.

Stevens, J. C., \& Marks, L. E. (1979). Spatial summation of cold. Physiology \& Behavior, 22, 541-547.

SZOLCś́NY1, J. (1977). A pharmacological approach to elucidation of the role of different nerve fibers and receptor endings in mediation of pain. Journal of Physiology, 73, 251-259.

SzolcsányI, J. (1982). Capsaicin-type pungent agents producing pyrexia. In A. S. Milton (Ed.), Handbook of experimental pharmacology (Vol. 60, pp. 437-478).

Szolcsányi, J. (1987). Capsaicin and nociception. Acta Physiologica (Hungary), 69, 323-332.

Szolcsányi, J., Anton, F., Reeh, P. W., \& Handwerker, H. O. (1988). Selective excitation by capsaicin of mechano-heat sensitive nociceptors in rat skin. Brain Research, 446, 262-268.

Toreguörk, H. E. (1974). Afferent $C$ units responding to mechanical, thermal and chemical stimuli in human non-glabrous skin. Acta Physiologica (Scandinavia), 92, 374-390.

TUCKETT, R. P. (1982). Itch evoked by electrical stimulation of the skin. Journal of Investigative Dermatology, 79, 368-373.

TUCKETT, R. P., \& WEI, J. Y. (1987). Responses to an itch-producing substance in the cat: II. Cutaneous receptor populations with unmyelinated axons. Brain Research, 413, 95-103.

van HeEs, J., \& GybeLS, J. (1981). C-nociceptor activity in human nerve during painful and non-painful skin stimulation. Journal of Neurophysiology, Neurosurgery and Psychiatry, 44, 600-607.

VERRILLO, R. T. (1966). Effect of spatial parameters on the vibrotactile threshold. Journal of Experimental Psychology, 71, 570-575.

(Manuscript received September 5, 1989; revision accepted for publication January 31,1990 .) 